\title{
The Structure of Gas-Phase Bradykinin Fragment 1-5 (RPPGF) Ions: An Ion Mobility Spectrometry and H/D Exchange Ion-Molecule Reaction Chemistry Study
}

\author{
Holly A. Sawyer, Joseph T. Marini, Earle G. Stone, Brandon T. Ruotolo, \\ Kent J. Gillig, and David H. Russell \\ Laboratory for Biological Mass Spectrometry, Department of Chemistry, Texas A\&M University, \\ College Station, Texas, USA
}

Ion mobility-mass spectrometry (IM-MS) data is interpreted as evidence that gas-phase bradykinin fragment 1-5 (BK1-5, RPPGF) $[\mathrm{M}+\mathrm{H}]^{+}$ions exist as three distinct structural forms, and the relative abundances of the structural forms depend on the solvent used to prepare the matrix-assisted laser desorption ionization (MALDI) samples. Samples prepared from organic rich solvents $(90 \%$ methanol $/ 10 \%$ water) yield ions having an ion mobility arrival-time distribution (ATD) that is dominated by a single peak; conversely, samples prepared using mostly aqueous solvents (10\% methanol/90\% water) yield an ATD composed of three distinct peaks. The BK1-5 $[\mathrm{M}+\mathrm{H}]^{+}$ions were also studied by gas-phase hydrogen/deuterium (H/D) exchange ion-molecule reactions and this data supports our interpretation of the IM-MS data. Plausible structures for BK1-5 ions were generated by molecular dynamics (MD). Candidate MD-generated structures correlated to measured cross-sections suggest a compact conformer containing a $\beta$-turn whereas a more extended, open form does not contain such an interaction. This study illustrates the importance of intra-molecular interactions in the stabilization of the gas-phase ions, and these results clearly illustrate that solution-phase parameters (i.e., MALDI sample preparation) greatly influence the structures of gas-phase ions. (J Am Soc Mass Spectrom 2005, 16, 893-905) (C) 2005 American Society for Mass Spectrometry

S tudies of peptide and protein structure play a major role in modern molecular biophysics and drive development of new, more specific and sensitive techniques. Gas-phase techniques for sequencing peptides and proteins are well established, and significant progress has been made in developing similar techniques for characterization of secondary $\left(2^{\circ}\right)$ and tertiary $\left(3^{\circ}\right)$ structures of gas-phase peptides/proteins [1-5]. Such studies increase our understanding of intra-molecular interactions and drive development of sequencing algorithms, but the relevance of gas-phase structure, structure in the absence of solvent [6], to solution-phase structure is still an open issue. The most important role of solvent is charge stabilization, and in the gas phase, charge stabilization occurs by intramolecular interactions [7]. Rapid progress is being made in the development of techniques that allow selective solvation (in terms of number of solvent molecules) of gas-phase peptide/protein ions [8], and these studies may serve to further our understanding of

Published online May 4, 2005

Address reprint requests to Dr. D. H. Russell, Laboratory for Biological Mass Spectrometry, Department of Chemistry, Texas A and M University, 3255 TAMU, College Station, TX 77843, USA. E-mail: russell@mail. chem.tamu.edu how solvent affects structure and function. In addition, gas-phase studies could enhance our understanding of intra-molecular interactions (i.e., hydrogen bonding, van der Waals interactions and hydrophobic forces), as well as how such interactions influence conformations in the solution phase or low dielectric environments (i.e., gas phase, membranes, and/or multimeric complexes).

Bradykinin (amino acid sequence RPPGFSPR) has been extensively studied using both experimental and computational techniques. Bradykinin is an excellent model peptide for structural studies because the presence of arginine $(\mathbf{R})$ at both the $\mathrm{N}$ - and C-terminus can potentially influence both structure and reactivity of the ion. For example, data from fragmentation studies were interpreted as evidence that intra-molecular hydrogen bonds exist between the $\mathrm{N}$ - and C-terminal arginine residues as well as between the serine (S) side-chain and the protonated guanidine group [9], but results from collision-induced dissociation (CID) studies emphasized the importance of interactions between the protonated guanidine group in bradykinin and $\mathbf{P}^{3}$ or $\mathbf{F}^{5}$ [10]. Gas-phase hydrogen/deuterium (H/D) exchange of bradykinin and derivatives of bradykinin indicate that the $[\mathrm{M}+\mathrm{H}]^{+}$ion might exist as a zwitterion [11], 
and this suggestion is supported by blackbody infrared dissociation (BIRD) studies of bradykinin and its methyl-ester derivative [12]. On the other hand, hydroiodic attachment studies suggest that the $[\mathrm{M}+\mathrm{H}]^{+}$ion does not exist as a zwitterion because of its capability to attach two HI molecules [13]. In spite of considerable research using a variety of experimental and computational tools there are key issues related to the structure of bradykinin ions that remain unresolved.

On the basis of ion mobility spectrometry (IMS) and molecular modeling studies, Bowers and coworkers suggested that protonated bradykinin $[\mathrm{M}+\mathrm{H}]^{+}$ions exist in multiple conformations having collision crosssections that differ by approximately two percent [5]. We previously studied bradykinin $[\mathrm{M}+\mathrm{H}]^{+}$ions using solvent dependent solution-phase $\mathrm{H} / \mathrm{D}$ exchange and circular dichroism (CD) and suggested that the structure(s) of bradykinin ions formed by MALDI depends upon the solvent conditions used to prepare the sample [14]. The H/D exchange data was interpreted as evidence that aqueous solvents favor formation of ions that lack any specific $2^{\circ}$ or $3^{\circ}$ structure, whereas organic solvents (trifluoroethanol, dioxane, and dimethyl sulfoxide) promote formation of compact conformers. We have begun a systematic study of bradykinin using IM-MS, gas-phase H/D exchange ion-molecule reactions, and molecular modeling to address the effects of sequence and solvent conditions used to prepare MALDI samples on the conformations of gas-phase ions. This paper focuses on bradykinin fragment 1-5 (RPPGF; denoted as BK1-5) $[\mathrm{M}+\mathrm{H}]^{+}$ions, and a follow-up paper will include other bradykinin fragments as well as a comparison of the $[\mathrm{M}+\mathrm{H}]^{+}$and $[\mathrm{M}$ $+\mathrm{Na}^{+}$ions. Data presented in this paper is used to establish that multiple structural features found in residues 1-5 (RPPGF) may contribute to the IMS peak broadening reported by Bowers and coworkers [5]. Of course, this approach eliminates effects of interactions involving residues 6-9 $\left(\mathbf{S}^{6}-\mathbf{P}^{7}-\mathbf{F}^{8}-\mathbf{R}^{9}\right)$, which have been implicated as potential intra-molecular interaction sites in previous solution-phase work [15]. These interactions will be explored further in future work. The effect of intra-molecular interactions on peptide structure and how these interactions change as a function of peptide sequence and solvent system used to prepare MALDI samples are also being pursued further.

\section{Experimental and Computational Methods}

Ion mobility measurements were made using a MALDIion mobility reflectron time-of-flight (TOF) mass spectrometer described previously [16]. MALDI was performed using a cartridge-type $\mathrm{N}_{2}$ laser $(337 \mathrm{~nm}$, Thermo Laser Science, Franklin, MA) operated at near-threshold laser powers. Laser power was controlled using a neutral density filter. Ion mobility measurements, arrival-time distributions (ATD) for the protonated molecule, $[\mathrm{M}+\mathrm{H}]^{+}$ions, were carried out using a 30.5 $\mathrm{cm}$ periodic-focusing drift cell operated at $\approx 1$ torr He at room temperature and using I $\mathrm{M}$ field strengths ranging from 20 to $30 \mathrm{~V} / \mathrm{cm}$.torr. It is important to note that both the laser power and the electric field-to- pressure ratio $(\mathrm{E} / \mathrm{p})$ affect the resolution of the ATDs (data not shown). That is, high laser powers result in broadening of the ATD for all species and at high E/p ratios the separation between the multiple conformations observed in the ATD of BK1-5 $[\mathrm{M}+\mathrm{H}]^{+}$ions decreases [17]. We interpret this data as evidence that at high laser powers and $\mathrm{E} / \mathrm{p}$ ratios the ion population sampled by IM-MS is no longer thermal; the ions have sufficient amounts of energy to undergo interconversion between conformers.

The mass spectrometer is a 2-stage reflectron TOF mass spectrometer with a path length of $\approx 1 \mathrm{~m}$. Ions formed by MALDI drift through the ion mobility (IM) cell, where they are separated on the basis of collision cross-section $(\Omega)$. The drift time of the ion through the cell depends on the strength of the applied electric field as well as the charge and collision cross-section of the ion. The mobility of the ion $(\mathrm{K})$ can be determined by measuring the drift velocity $\left(\mathrm{v}_{\mathrm{d}}\right)$ :

$$
\mathrm{K}=\frac{\mathrm{v}_{\mathrm{d}}}{E}=\frac{L}{t_{d} E}
$$

[16], where $t_{d}$ is the drift time, $L$ is the drift length and $E$ is the applied electric field [18]. By approximating the ion and buffer gas as hard spheres, the collision crosssection can be calculated from the mobility $(\mathrm{K})$, temperature $(\mathrm{T})$, pressure $(\mathrm{P})$, number density $\left(\mathrm{N}^{*}\right)$, masses of both the ion $\left(\mathrm{m}_{\mathrm{I}}\right)$ and the buffer gas $\left(\mathrm{m}_{\mathrm{b}}\right)$, and the charge of the ion $(\mathrm{z})$ :

$$
\Omega=\frac{(18 \pi)^{\frac{1}{2}}}{16} \frac{\mathrm{ze}}{\left(k_{\mathrm{B}} \mathrm{T}\right)^{\frac{1}{2}}}\left[\frac{1}{\mathrm{~m}_{\mathrm{I}}}+\frac{1}{\mathrm{~m}_{\mathrm{B}}}\right]^{\frac{1}{2}} \frac{1}{\mathrm{~K}} \frac{760}{\mathrm{P}} \frac{\mathrm{T}}{273.15} \frac{1}{\mathrm{~N}^{*}}
$$

[17], where $e$ is ionic charge and $k_{B}$ is Boltzmann's constant. The collision cross-sections reported herein were obtained using an internal calibration method [19]. Briefly, a peptide with a known cross-section (bradykinin) is used as the standard and the difference between the drift times of the standard and the ion of interest is measured multiple times and is used to calculate the $\Omega$ of the ion of interest relative to the standard. The ATDs presented in this paper were acquired by selecting a narrow mass range from the two-dimensional (mass and mobility spectra) data and looking at it in one dimension (mobility spectrum).

Ion mobility-surface-induced dissociation mass spectrometry (IM-SID-MS) experiments were performed as described previously [20]. Briefly, ions formed by MALDI and separated using an ion mobility cell are dissociated by energetic $(40 \mathrm{eV})$ collisions with a fluorinated self-assembled monolayer (F-SAM) surface. The 
fragment ions formed by SID are mass analyzed in a linear TOF mass spectrometer.

Gas-phase H/D exchange ion-molecule reactions were carried out using a 3 tesla Fourier transform (FT) ion cyclotron resonance (ICR) mass spectrometer [21]. Ions formed by MALDI (355 nm from a Nd:YAG laser; 600 ps pulse duration) were trapped in the source region of a two-section cylindrical $(4.0 \mathrm{~cm} \times 4.0 \mathrm{~cm})$ ion cell, which was held a constant pressure $\left(0.6-1 \times 10^{-7}\right.$ torr) of $\mathrm{ND}_{3}$ or deuterated acetic acid $\left(\mathrm{CD}_{3} \mathrm{CO}_{2} \mathrm{D}\right)$, and the reaction products formed over times ranging from 0.005 to $360 \mathrm{~s}$ were monitored. Temporal plots were generated by plotting ion intensity, corrected for the natural isotope abundance, versus time.

All molecular dynamics calculations were performed with the Cerius (Accelrys) suite of programs using the consistent force field (CFF1.01). Model structures for the peptide ions were generated using simulated annealing cycles $[22,23]$ where the structure was heated from 300 Kelvin $(\mathrm{K})$ to $1000 \mathrm{~K}$ in steps of $50 \mathrm{~K}$ with relaxation times of 0.1 picoseconds (ps), making the entire simulation time 280 ps for 100 cycles. Semi-empirical calculations using Austin model (AM1) parameters were used to determine the energy of each structure. The collision cross-sections of each structure were calculated using the projection approximation, the exact hard sphere scattering method and the trajectory method using MOBCAL, a program designed by Mesleh et al. $[24,25]$. Four hundred structures of BK1-5 were generated by simulated annealing. Two hundred low-energy (within $15 \mathrm{kcal} / \mathrm{mol}$ of the lowest energy structure) structures were classified into groups based on structure and intra-molecular interactions. The structures proposed herein were chosen based on agreement between the experimental collision cross-section, cluster population (structures in the most populated cluster within the cross-section range were considered most likely) as well as energy level.

The peptides used in these studies were purchased from Sigma Aldrich (St. Louis, MO) or SynPep (Dublin, CA) and used without further purification. The compounds used as MALDI matrices, 2,5-dihydroxybenzoic acid, 2, 4, 6-trihydroxyacetophenone, p-nitroaniline, 3-nitrobenzyl alcohol, glycerol, and $\mathrm{D}(-)$ fructose were purchased from Sigma Aldrich and used without further purification. $\alpha$-Cyano- 4 hydroxycinnamic acid was purchased from Sigma Aldrich and was recrystallized prior to use. $\mathrm{ND}_{3}$ gas was purchased from Bachem (Torrance, CA) and used without filtering. HPLC grade methanol and deionized (Milli-Q Water System, Millipore, Billerica, MA) water were used for all experiments. The MALDI samples used for IM-MS measurements were prepared using the dried droplet method with $\alpha$-cyano- 4 hydroxycinnamic acid as matrix with a matrix-to-analyte molar ratio of 400:1. Liquid MALDI [26] samples for IM-MS were prepared by applying the liquid matrix (3-nitrobenzyl alcohol and/or glycerol) to the probe tip and then adding $\sim 1 \mu \mathrm{L}$ of a solution containing the peptide (in methanol) at approximately 1 $\mathrm{mg} / \mathrm{mL}$. In some cases additional matrix material was used (p-nitroaniline or $\alpha$-cyano- 4 hydroxycinnamic acid, in methanol), and in such cases, the peptidematrix mixture with a matrix-to-analyte molar ratio of 400:1 was added to the liquid matrix (glycerol). Samples used for H/D exchange studies were prepared using the same general methods. The use of $\mathrm{D}(-)$ fructose for reducing the internal energies of the ions formed by MALDI has been previously described [27], and we observed similar behavior for $\mathrm{H} / \mathrm{D}$ exchange reaction chemistry. That is, without added fructose the ions appear to have a broad range of internal energies. Additionally, sample consumption was increased, prohibiting observation of the full time range without replenishing the sample, which would result in large fluctuations of the deuterating agent pressure.

\section{Results and Discussion}

Bowers and coworkers used IM-MS to investigate the structure of gas-phase bradykinin $[\mathrm{M}+\mathrm{H}]^{+}$ions, and they concluded that the collision cross-sections of the different conformers of bradykinin $[\mathrm{M}+\mathrm{H}]^{+}$ions are very similar and cannot be separated or that the conformers interconvert on the timescale of the experiment [5]. We have designed a series of experiments to reexamine the structure of bradykinin gas-phase ions; however, our approach is to study specific fragments of bradykinin in an effort to deconvolute the effect of specific amino acid residues on the gas-phase conformation of bradykinin; a similar approach was used by London et al. in their NMR studies of solution-phase bradykinin [28]. Here we report ATD data for bradykinin fragment $1-5$, measured using IM-MS, and proposed structures for the ions presented herein were derived by matching the experimentally measured collision cross-section with the calculated collision crosssection for structures obtained by using molecular dynamics. We also measured the rates for the first $H / D$ exchange reaction and the overall extent (total number of exchanges) of $\mathrm{H} / \mathrm{D}$ exchange. We focus our $\mathrm{H} / \mathrm{D}$ exchange studies on the first exchange reaction because of concerns that structural rearrangement reactions of the initially formed BK1-5 ions may occur during the lifetime of the ion-molecule collision complex [8], i.e., time that solvent (H/D reagent) interacts with the ion. In particular, gas-phase $\mathrm{H} / \mathrm{D}$ exchange reactions of $\mathrm{ND}_{3}$ occur through the "onium" ion mechanism, in which $\mathrm{ND}_{3}$ extracts a proton from the peptide and the resulting complex is then solvated by the peptide [29]. Facile exchange occurs when the onium ion is stabilized by hydrogen bonding interactions with the peptide [30]. Campbell et al. observed less exchange for collisionally activated ions, which they attributed to a reduction in the lifetime of the hydrogen-bonded collision complex [29]. In addition, Wyttenback and Bowers reported a long-lived $\mathrm{D}_{2} \mathrm{O}$-bradykinin $[\mathrm{M}+\mathrm{H}]^{+}$ion complex $(>1$ ns) [31]. Long-lived ion-neutral interactions could obscure subtle differences in the structures of the reacting 

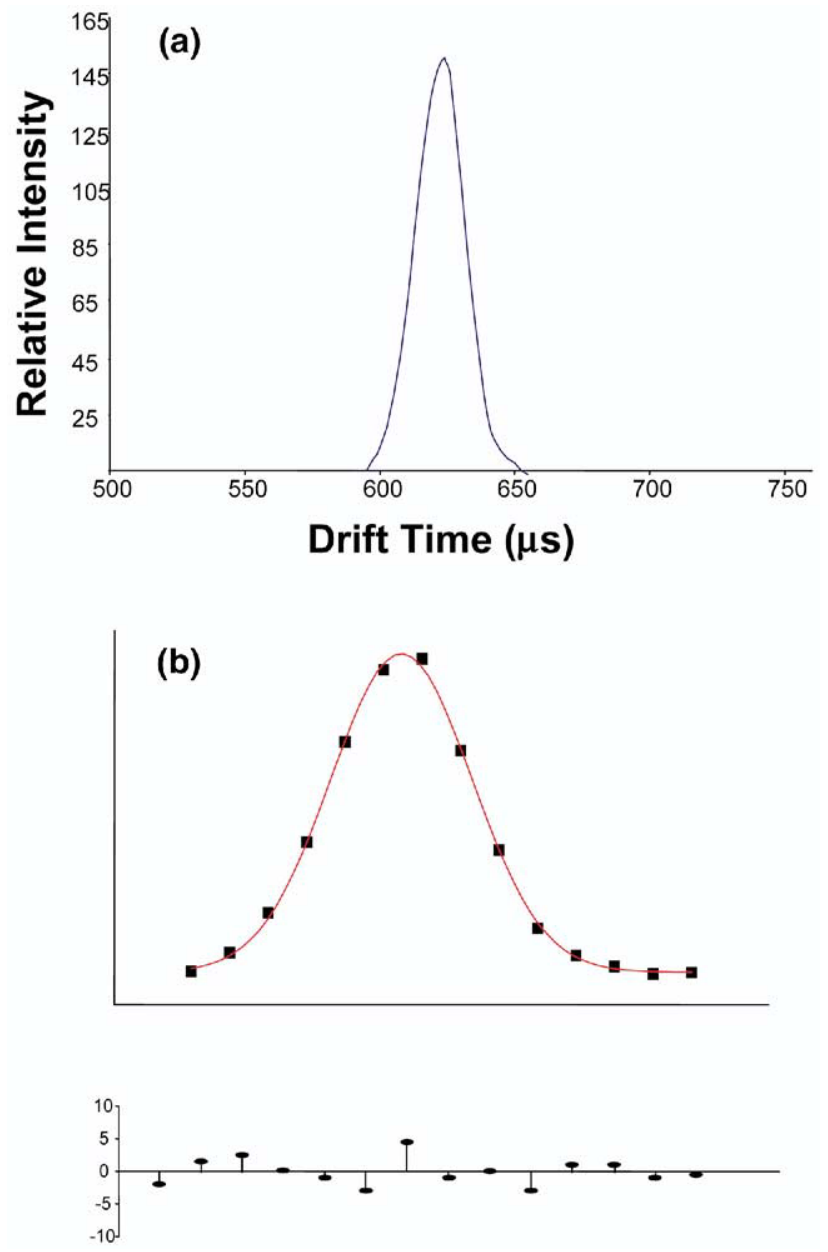

Figure 1. (a) ATD of BK2-7 $[\mathrm{M}+\mathrm{H}]^{+}$ions. (b) Best fit and residual of the ATD of BK2-7 $\left[\mathrm{M}+\mathrm{H}^{+}\right.$ions.

ion, thus the rate of the first exchange with reagents such as $\mathrm{CD}_{3} \mathrm{CO}_{2} \mathrm{D}$ should provide a better measure of the accessibility of the exchangeable $\mathrm{H}$ to solvent, which we then use to infer structural differences in the ion prior to interaction with the "solvent."

The ATDs of bradykinin and BK1-8 (RPPGFSPF; $m / z$ 905), BK2-9 (PPGFSPFR; $m / z$ 905), and BK5-9 (FSPFR; $\mathrm{m} / \mathrm{z}$ 653.8) are dominated by broad, unresolved peaks (data not shown), whereas the ATD of BK2-7 (PPGFSP; $\mathrm{m} / \mathrm{z} 601.7$ ) is dominated by a single, relatively narrow, peak (Figure 1a). Conversely, multiple peak features are observed in the ATD of bradykinin analogs that contain an N-terminal arginine residue, viz. BK1-5 (RPPGF; $m / z$ 573.7), BK1-6 (RPPGFS; $m / z$ 660.7), and BK1-7 (RPPSFSP; $m / z$ 757.9), and in the case of BK1-5 the ATD is composed of three distinct peaks (see Figure 2a). Counterman et al. previously reported multiple peak features in the ATD of $42 \%$ of proline containing tryptic peptides composed of 10 or fewer residues, for which they suggested that the cis and trans configurations of proline result in structural isomers with different collision cross-sections [4]. We do not feel that the different peaks in the ATD of BK1-5 are due solely to the presence of proline, because we do not see multiple peaks for BK1-8, BK2-7, BK5-9, or bradykinin. A more detailed discussion of the structural assignments is presented in a later section of this paper. IM-SIDTOF-MS was used to verify that each of the ion mobility-separated ion signals is due to BK1-5 [M + $\mathrm{H}]^{+}$ions as opposed to background ions or another charge state of BK1-5. For example, SID spectra were recorded at specific points in the ATD for the BK1-5 [M $+\mathrm{H}]^{+}$ions, and two examples are shown in Figure 3. In each of the SID spectra the fragment ions that are detected are identical, which we interpret as evidence that the peaks in the ATD arise from two different conformers, or populations of conformers, of the BK1-5 $[\mathrm{M}+\mathrm{H}]^{+}$ions. In particular, the relative abundance of the signals for sequence informative fragment ions $\left(b_{i}\right.$ and $y_{j}$ ) are very similar in both spectra, and the spectra compare very well to CID spectra (data not shown) taken using a conventional TOF-TOF (ABI 4700 Proteomics Analyzer) instrument [17]. Note also that signals formed by neutral loss (loss of $\mathrm{NH}_{3}$ and loss of the C-terminal phenylalanine $\left[b_{4}+\mathrm{H}_{2} \mathrm{O}\right]$ ) are almost identical in the two spectra, and the same can be said about the immonium ions and internal cleavage (PP and PG fragment ions) product ions.

Although we attribute the multiple peaks in the ATD of BK1-5 $[\mathrm{M}+\mathrm{H}]^{+}$ions to the presence of multiple conformers of the $[\mathrm{M}+\mathrm{H}]^{+}$ions, we also evaluated several other possibilities. For example, dissociation of higher-mass species (i.e., matrix clusters of BK1-5 [M + $\mathrm{H}^{+}$ions or multimers of BK1-5 [32]) to the $\mathrm{m} / z 573$ signal could lead to an appearance of multiple peaks in the ATD of BK1-5 [M $+\mathrm{H}]^{+}$ions [32]. However, the SID data (vida supra) are inconsistent with this explanation due to the similarity in the spectra (relative abundance and observed fragment ions) for both IM resolved species (Figure 3). If the ATD were composed of dissociation products of higher mass species, we would expect the relative fragment ion abundance to be different, because the relative energies required to fragment a multimer versus a monomer are different [17]. Reactions with background gases, i.e., residual $\mathrm{H}_{2} \mathrm{O}$, in the drift tube could potentially lead to the appearance of multiple peaks in the ATD of BK1-5 $[\mathrm{M}+\mathrm{H}]^{+}$ions. However, the ATD of BK2-7 $[\mathrm{M}+\mathrm{H}]^{+}$ions (which has 4 residues in common with BK1-5 $[\mathrm{M}+\mathrm{H}]^{+}$ions) is a single narrow Gaussian curve (Figure 1a and b). Owing to the sequence homology between BK2-7 and BK1-5, it seems unlikely that the reaction chemistry of these two ions would differ significantly from that of BK2-7 $[\mathrm{M}+\mathrm{H}]^{+}$ ions. Furthermore, the ATD of BK1-5 $[\mathrm{M}+\mathrm{H}]^{+}$ions is composed of multiple peaks only when MALDI samples are prepared with aqueous solvents (vida infra), which should not affect drift tube reaction chemistry or the fragment ion spectra.

We also examined the effect of solvent composition on the ATD of BK1-5 $[\mathrm{M}+\mathrm{H}]^{+}$ions. For example, MALDI sample deposits were prepared from solutions composed of $100 \%$ methanol, $75 / 25 \%$ (vol/vol) metha- 

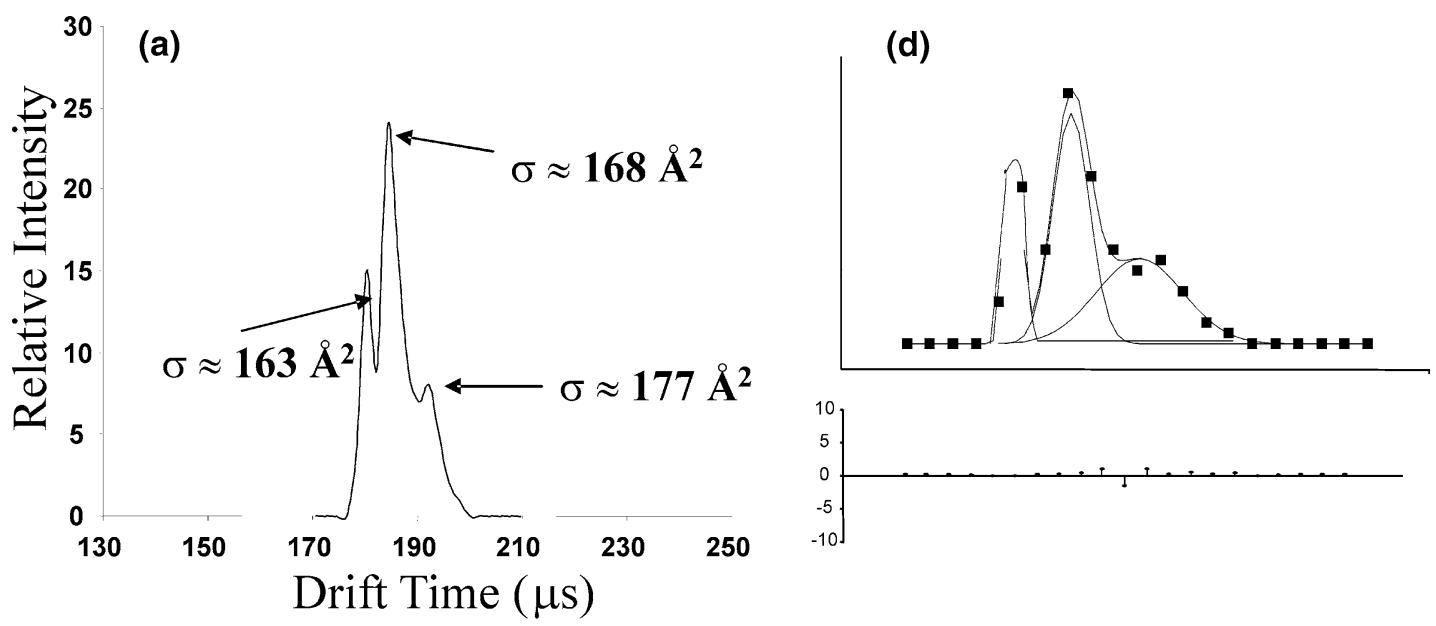

60

(b)

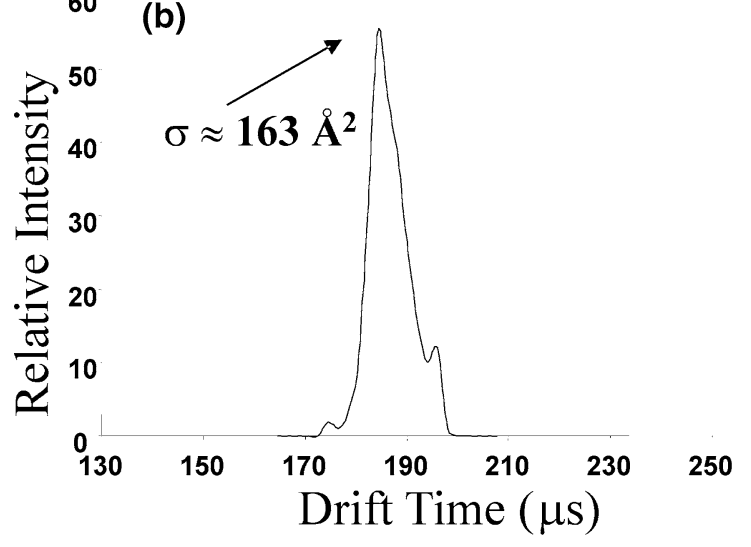

(e)
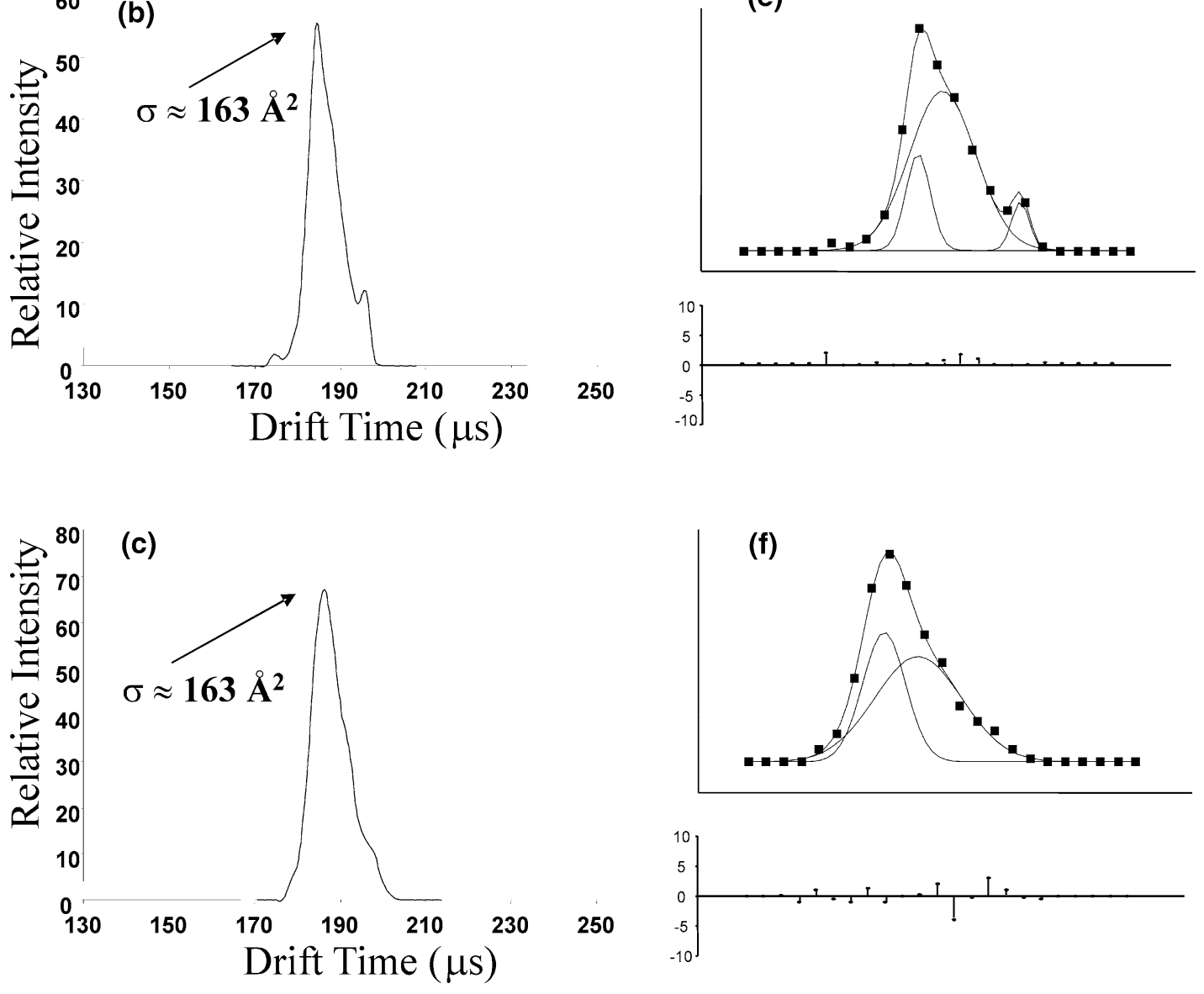

Figure 2. ATDs and best fits of the ATDs of $[\mathrm{M}+\mathrm{H}]^{+}$ions of BK1-5 ionized from starting solvent systems composed varying percents of $\mathrm{H}_{2} \mathrm{O}$ methanol. Below the best fits are the residuals between the data and the fit. The individual Gaussian curves and the total fit of the ATD are shown. (a) $10 \%$ Methanol, 90\% $\mathrm{H}_{2} \mathrm{O}$; (b) 75\% methanol, $25 \% \mathrm{H}_{2} \mathrm{O}$; (c) 100\% methanol; (d) best fit and residual of ATD of $[\mathrm{M}+\mathrm{H}]^{+}$ions from starting solvent system composed of $10 \%$ methanol, $90 \% \mathrm{H} 2 \mathrm{O}$; (e) best fit and residual of ATD of $[\mathrm{M}+\mathrm{H}]^{+}$ions from starting solvent system composed of $75 \%$ methanol, $25 \% \mathrm{H} 2 \mathrm{O}$; (f) best fit and residual of ATD of $[\mathrm{M}+\mathrm{H}]^{+}$ions from starting solvent system composed of $100 \%$ methanol.

nol/water, $50 / 50 \%$ (vol/vol) methanol/water, $25 / 75 \%$ (vol/vol) methanol/water, and $10 / 90 \%$ (vol/vol) methanol/water. The ATD for samples prepared using $100 \%$ methanol is composed of a broad, single maxima (see

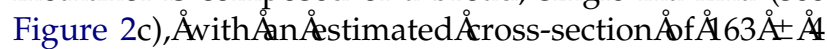
$\AA^{2}$, which is identical to that of the first peak in the 


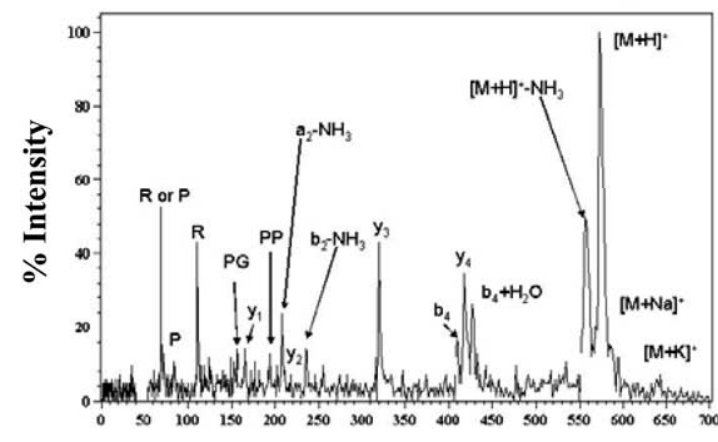

(a)

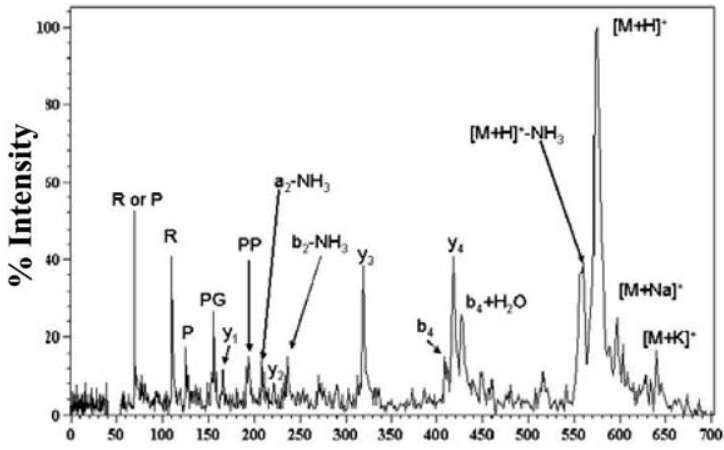

(b) $\mathbf{m} / \mathbf{z}$

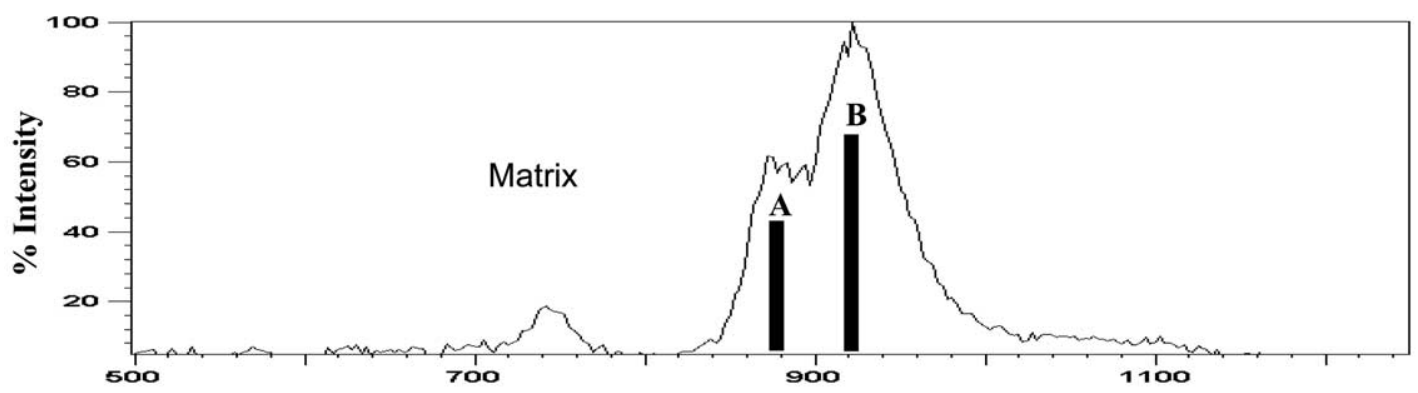

(c)

Drift Time ( $\mu$ sec)

Figure 3. Ion mobility-surface induced dissociation spectra of $[\mathrm{M}+\mathrm{H}]+$ ions of BK1-5. (a) Surface induced dissociation spectrum of ions found in time slice A [see (c)]. (b) Surface induced dissociation spectrum of ions found in time slide B [see (c)]. (c) ATD of BK1-5 as observed in the ion mobility-surface induced dissociation experiment. The time slices of ions that were collided on the surface to produce the spectra [see (a) and (b)] are shown with dark lines and labeled A and B.

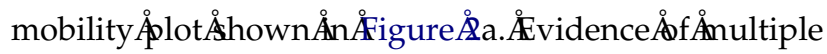
conformations (i.e., broad peaks, shoulders or multiple peaks) $[4]$ [ methanol solvent, demonstrating that more aqueous solvent conditions favor formation of BK1-5 $[\mathrm{M}+\mathrm{H}]^{+}$ ions which have larger cross-sections. The ATD changes dramatically at $10 \% / 90 \%$ methanol/water, and under these conditions the more abundant conformer has a collision cross-section of $168 \pm 5 \AA^{2}$. Note the small shoulder on the ATD for ions formed at $10 \% / 90 \%$ methanol/water, which we interpret as the presence of a third conformer having a cross-section of $177 \pm 3 \AA^{2}$.

The ATDs of all the bradykinin analogs were analyzed using a Gaussian curve fitting program (OriginLab Corporation, Northampton, MA). Such curve fitting routines are only used to extract qualitative information from the data, and a more critical treatment aimed at extracting more quantitative peak shape information will be described in a future paper. While a single Gaussian curve accurately $\left(\mathrm{R}^{2}=0.998, \chi^{2}=5.49\right)$

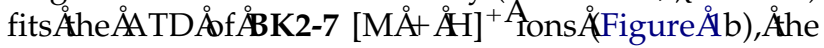
ATDs of BK1-5 $[\mathrm{M}+\mathrm{H}]^{+}$ions generated from MALDI samples prepared in $100 \%$ methanol, $75 \%$ methanol, and $90 \%$ water are best fit with multiple Gaussian curves. Note that the ATD of BK1-5 $[\mathrm{M}+\mathrm{H}]^{+}$ions generated from MALDI samples prepared in 100\% methanol Ås ominated

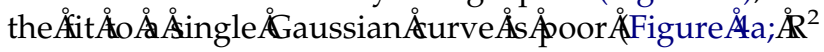

$\left.=0.965, \chi^{2}=18.7\right)$, with residuals ranging between +8

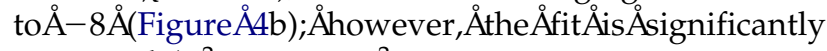
improved $\left(R^{2}=0.997, \chi^{2}=1.89\right)$ if 2 Gaussian curves

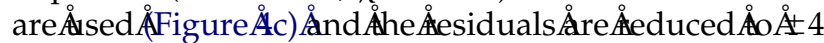
(Figure A4d).ÅTheÅfitÅof ÅtheÅdataÅis Åonly Åmarginally improved $\left(R^{2}=0.999, \chi^{2}=0.77\right)$ if 3 Gaussian curves

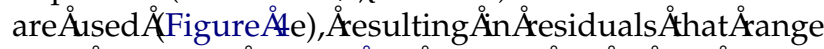

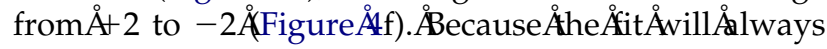
improve by increasing the number of Gaussian curves used, the criteria we used to estimate the "best fit" is the number of Gaussian curves, N, that most improve the fit over $\mathrm{N}-1$ Gaussian curves and resulted in little to no improvement of the fit is found for $\mathrm{N}+1$ curves. By using similar methods and logic we determined that both ATDs of BK1-5 $[\mathrm{M}+\mathrm{H}]^{+}$ions generated from MALDI samples prepared in $75 \%$ methanol and $90 \%$ water are best fit using three Gaussian curves $\left(\mathrm{R}^{2}=\right.$ 0.999, $\chi^{2}=0.48$ and $\mathrm{R}^{2}=0.997, \chi^{2}=0.24$, respectively). The best fits of the ATDs of BK1-5 $[\mathrm{M}+\mathrm{H}]^{+}$ions generated from MALDI samples prepared in 100\% methanol, $75 \%$ methanol, and $90 \%$ water are shown in

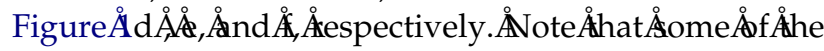
decomposed peaks are broader (FWHM) than others and we assume this indicates a population of conformers that have very similar (within $2 \%$ ) collision crosssections, which was addressed previously in the Experimental and Computational Methods section.

ATD for BK1-5 [M $+\mathrm{H}]^{+}$ions generated from liquid 


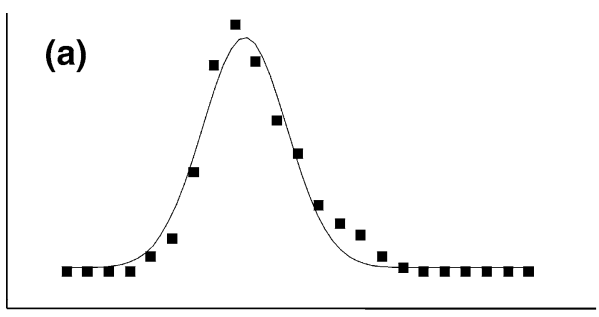

(b)
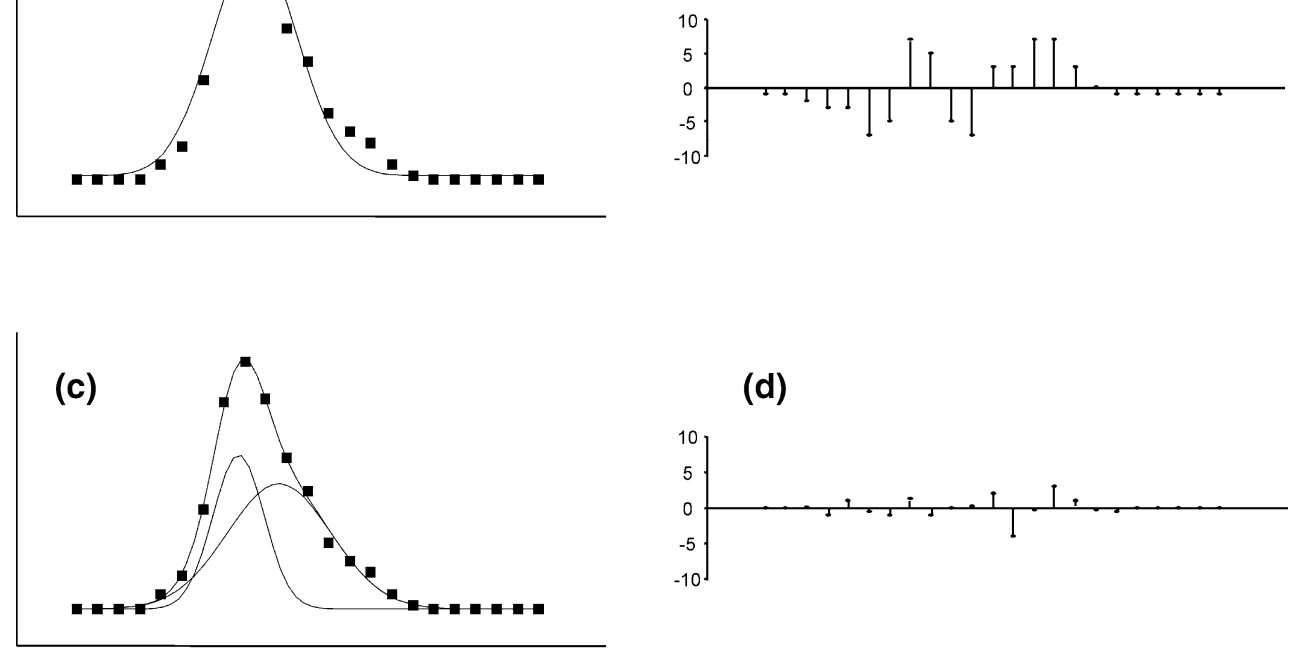

(d)
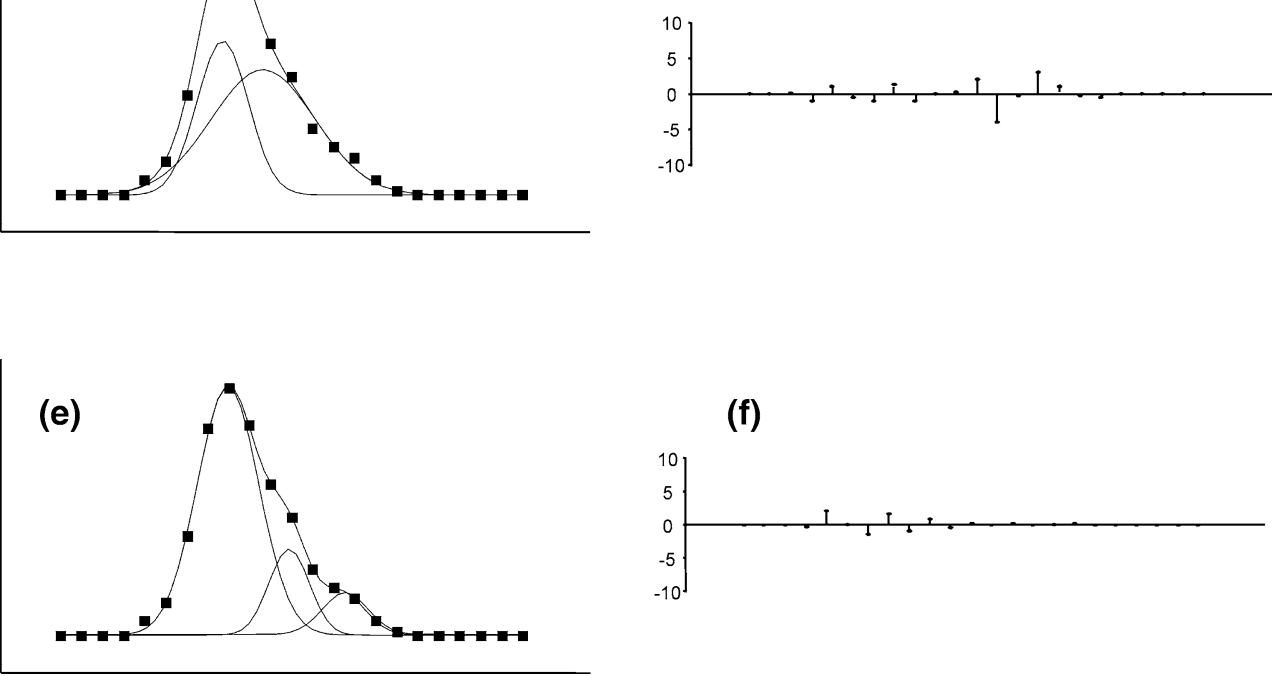

(f)

Figure 4. Fits and residuals of the ATD of BK1-5 $[\mathrm{M}+\mathrm{H}]^{+}$ions generated from MALDI samples prepared in $100 \%$ methanol. (a) Fit of 1 Gaussian curve to the ATD of BK1-5 [M $+\mathrm{H}]^{+}$ions generated from MALDI samples prepared in 100\% methanol; (b) residual differences between the data and the fit shown in (a); (c) Fit of 2 Gaussian curves to the ATD of BK1-5 $[\mathrm{M}+\mathrm{H}]^{+}$ions generated from MALDI samples prepared in $100 \%$ methanol; (d) residual differences between the data and the fit shown in (c); (e) Fit of 3 Gaussian curves to the ATD of BK1-5 $[\mathrm{M}+\mathrm{H}]^{+}$ions generated from MALDI samples prepared in $100 \%$ methanol; (f) Plotted residual differences between the data and the fit shown in (e).

matrices were also measured in order to compare the effects of desorption from dried droplet sample preparations versus liquid matrices on the ATD (i.e., gasphase ion structure) (data not shown). The ATD of BK1-5 $[\mathrm{M}+\mathrm{H}]^{+}$ions generated from neat 3-nitrobenzyl alcohol, which has a relatively strong absorption at $355 \mathrm{~nm}$ and can act as a MALDI matrix, containing residual amounts of methanol (from the peptide solution, see Experimental and Computational Methods section), contained a single peak corresponding to a collision cross section of $165 \AA^{2}$. Similarly, the ATD of BK1-5 $[\mathrm{M}+\mathrm{H}]^{+}$ions generated from 3-nitrobenzyl alcohol and glycerol, again with residual amounts of methanol from the peptide solution (see Experimental and Computational Methods section) also contained a single peak corresponding to a collision cross-section of $163 \AA^{2}$. Note that these values are similar to that for BK1-5 $[\mathrm{M}+\mathrm{H}]^{+}$ions generated from dried droplet MALDI samples prepared from methanolic solvents $\left(163 \AA^{2}\right)$. Conversely, ATDs of BK1-5 $[\mathrm{M}+\mathrm{H}]^{+}$ions generated from glycerol (a "water-like" solvent), which contains small amounts of MALDI matrix (either pnitroaniline or $\alpha$-cyano- 4 hydroxycinnamic acid), contain multiple peaks, similar to BK1-5 $[\mathrm{M}+\mathrm{H}]^{+}$ions generated from dried droplet MALDI samples prepared from aqueous solutions, and the cross-sections of the ions are estimated to be $161 \AA^{2}, 167 \AA^{2}$, and $173 \AA^{2}$, values that are very similar to those obtained using dried droplet MALDI samples $\left(163,168\right.$, and $\left.177 \AA^{2}\right)$.

$\mathrm{H} / \mathrm{D}$ exchange reactions of BK1-5 $\left[\mathrm{M}+\mathrm{H}^{+}\right.$ions with $\mathrm{ND}_{3}$ and deuterated acetic acid $\left(\mathrm{CD}_{3} \mathrm{CO}_{2} \mathrm{D}\right)$ were also used as structural probes. Deuterated ammonia is an excellent $\mathrm{H} / \mathrm{D}$ exchange reagent because the gasphase basicity of $\left.\mathrm{ND}_{3 \AA}(204.0 \AA \mathrm{kcal} / \mathrm{mol}) \AA 29\right]$ promotes rapid $H / D$ exchange, thus $N_{3}$ is a useful reagent for

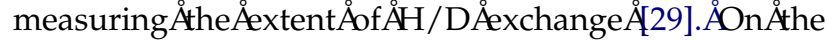
other hand, because we are primarily interested in using $\mathrm{H} / \mathrm{D}$ reactions to differentiate subtle differences in the structural composition of the ion population, all kinetic measurements are based on reactions using 


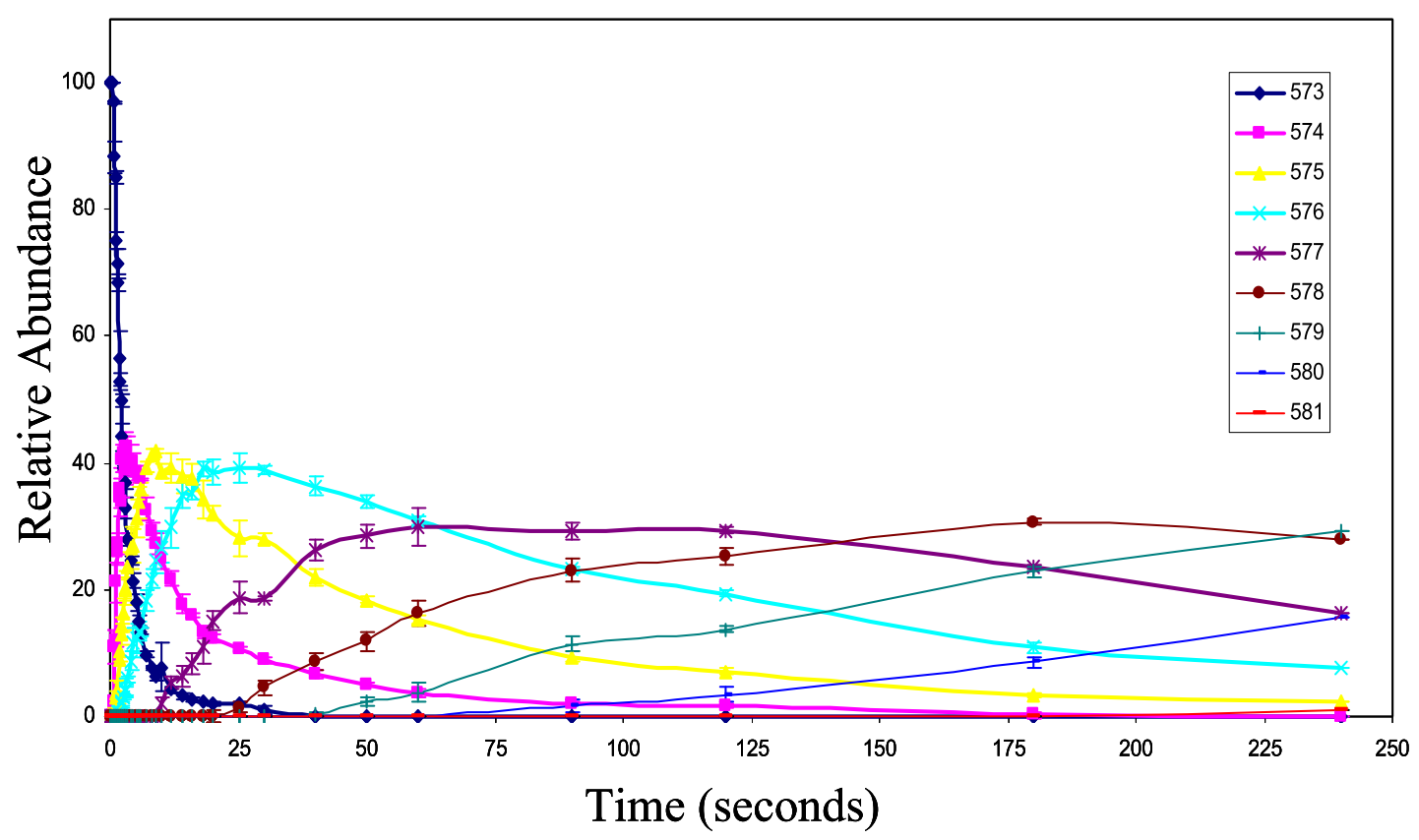

Figure 5. Temporal plot of the gas-phase H/D exchange of $[\mathrm{M}+\mathrm{H}]^{+}$ions of BK1-5 ionized from aqueous solvent systems.

deuterated acetic acid $\left(\mathrm{CD}_{3} \mathrm{CO}_{2} \mathrm{D}\right.$; gas phase basicity

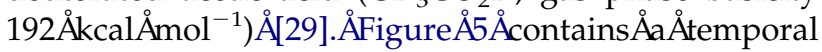
profile for $\mathrm{H} / \mathrm{D}$ exchange of BK1-5 $[\mathrm{M}+\mathrm{H}]^{+}$ions with $\mathrm{ND}_{3}$ generated from aqueous containing solvent systems. Rate constants for the first exchange of BK1-5 [M $+\mathrm{H}]^{+}$ions with $\mathrm{ND}_{3}$ and $\mathrm{CD}_{3} \mathrm{CO}_{2} \mathrm{D}$ were obtained by plotting the natural $\log$ of the ion abundance as a function of reaction time at a constant pressure of H/D exchange reagent. These plots should generate a straight line and the slope of the curve can be used to calculate the rate of exchange. Curvature (nonlinearity) in the plot may be observed if the ion population is composed of ions of different structures having different reactivities (accessibilities by the reacting neutral). It appears that a large fraction of the population of BK1-5 $[\mathrm{M}+\mathrm{H}]^{+}$ions generated from aqueous solvent systems areÅveryÅreactiveÅ(seeÅFigureÅba);Åhowever, Åas Åthe reactive ion population is depleted, the slope of the decay curve changes because the remaining ions are less reactive. The biexponential decay is more pronounced in the log plots obtained using $\mathrm{CD}_{3} \mathrm{CO}_{2} \mathrm{D}$ (see

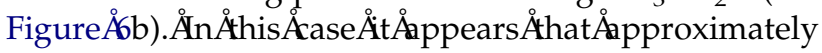
$70 \%$ of the total ion abundance is a fast-exchanging conformer, and the remaining $30 \%$ is composed of a slow-exchanging conformer. The decay curve for $\mathrm{m} / \mathrm{z}$ 573 can be fit using both a biexponential $(\mathrm{R}=0.998)$ and a single exponential $(R=0.947)$ decay equation, but a biexponential decay is a significantly better fit. We interpret the observation of two distinct decay rates of the $\mathrm{H} / \mathrm{D}$ exchange as evidence of multiple (at least two) reactive structures of BK1-5 $[\mathrm{M}+\mathrm{H}]^{+}$ions. Additionally, the rate plots indicate a similar relative population of the two conformers to that observed in the IM experiments.
Gas-phase H/D exchange reactions were also performed on BK1-5 $[\mathrm{M}+\mathrm{H}]^{+}$ions prepared from solvent

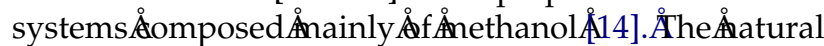
log plot of BK1-5 $[\mathrm{M}+\mathrm{H}]^{+}$ions generated from these MALDI sample solutions yields a straight line (see

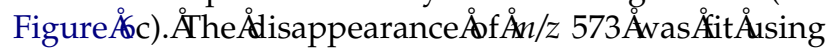
both a biexponential and single exponential decay and both fit the trend equally well $(R=0.994)$, therefore a single exponential fit was accepted. Additionally, the rate of reaction of BK1-5 [M+H] ${ }^{+}$ions generated from methanolic MALDI solvents is very similar to that of a large fraction of the ion population of BK1-5 from aqueous solvents.

BK2-7 was also investigated with $\mathrm{H} / \mathrm{D}$ exchange (data not shown). The natural log plot of BK2-7 yields a reasonably straight line $(\mathrm{R}=0.951)$. Both a biexponential and a single exponential decay fit the data relatively well ( $\mathrm{R}=0.976$ for both); therefore a single exponential fit was accepted. We interpret the IM-MS and H/D exchange data of BK2-7 to suggest the presence of one conformer for the $[\mathrm{M}+\mathrm{H}]^{+}$ions of BK2-7.

IM-MS and gas-phase H/D exchange can be used to investigate the gas-phase conformation of biomolecules to a limited level of certainty; however, such experimental information can then be used along with MD data to address specific structural issues (i.e., helical versus globular structure, intra-molecular interactions, charge-stabilization, or salt bridge interactions). MD, AM1 and density functional theory (DFT) energy calculations, and filtering techniques were used to generate and select possible conformations for BK1-5.

A plausible structure $\left(\Omega_{\text {theo }}=163 \AA^{2}\right)$ for the compact conformation found in organic solvents is shown

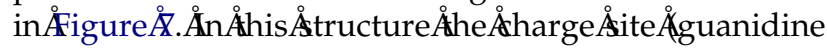



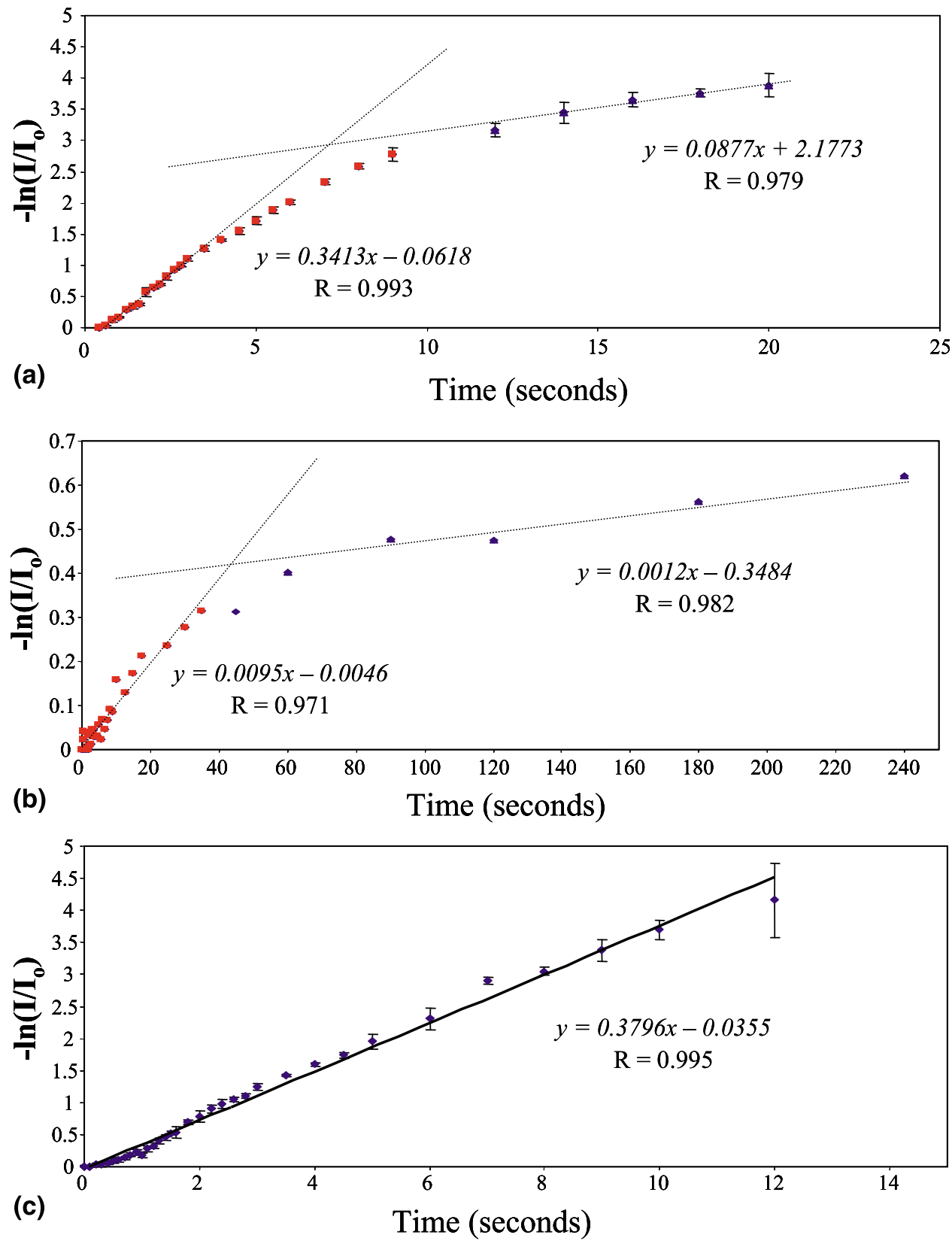

Figure 6. (a) Inverse log plot (rate plot) of the $[\mathrm{M}+\mathrm{H}]^{+}$ions of BK1-5 ionized from aqueous solvent systems undergoing gas-phase H/D exchange with ND3. (b) Inverse log plot (rate plot) of the [M + $\mathrm{H}]+$ ions of BK1-5 ionized from aqueous solvent systems undergoing gas-phase H/D exchange with deuterated acetic acid. (c) Inverse log plot (rate plot) of the $[\mathrm{M}+\mathrm{H}]{ }^{+}$ions of BK1-5 ionized from organic solvent systems undergoing gas-phase $\mathrm{H} / \mathrm{D}$ exchange with $\mathrm{ND}_{3}$.

group of arginine) is in close proximity to several less basic sites (C-terminus and backbone carbonyl oxygen atoms), and this interaction is expected to stabilize the onium ion during the $\mathrm{H} / \mathrm{D}$ exchange ion-molecule reaction allowing exchange to take place. The guanidine group is solvated by carbonyl oxygen atoms, however it is still accessible to $\mathrm{ND}_{3}$, allowing facile $\mathrm{H} / \mathrm{D}$ exchange, making this conformer consistent with the results of the $\mathrm{H} / \mathrm{D}$ exchange ion-molecule reactions. This conformation is also consistent with expectations for conformations generated from organic solutions because the hydrophilic sites are turned inward to interact with each other, which would allow the hydrophobic sites to

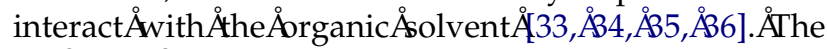
pro $^{2}$ - pro $^{3}$ bond in this structure is in the cis form, and this configuration promotes a $\beta$-turn interaction, which agrees with the assignment of a cis configuration being

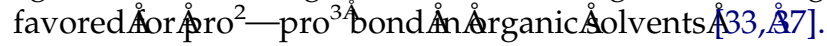
$\beta$-turn interactions for bradykinin fragments $1-4$ and 1-5 in organic solvent systems were previously ob-

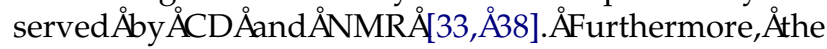
charged guanidine group in the proposed structure for 


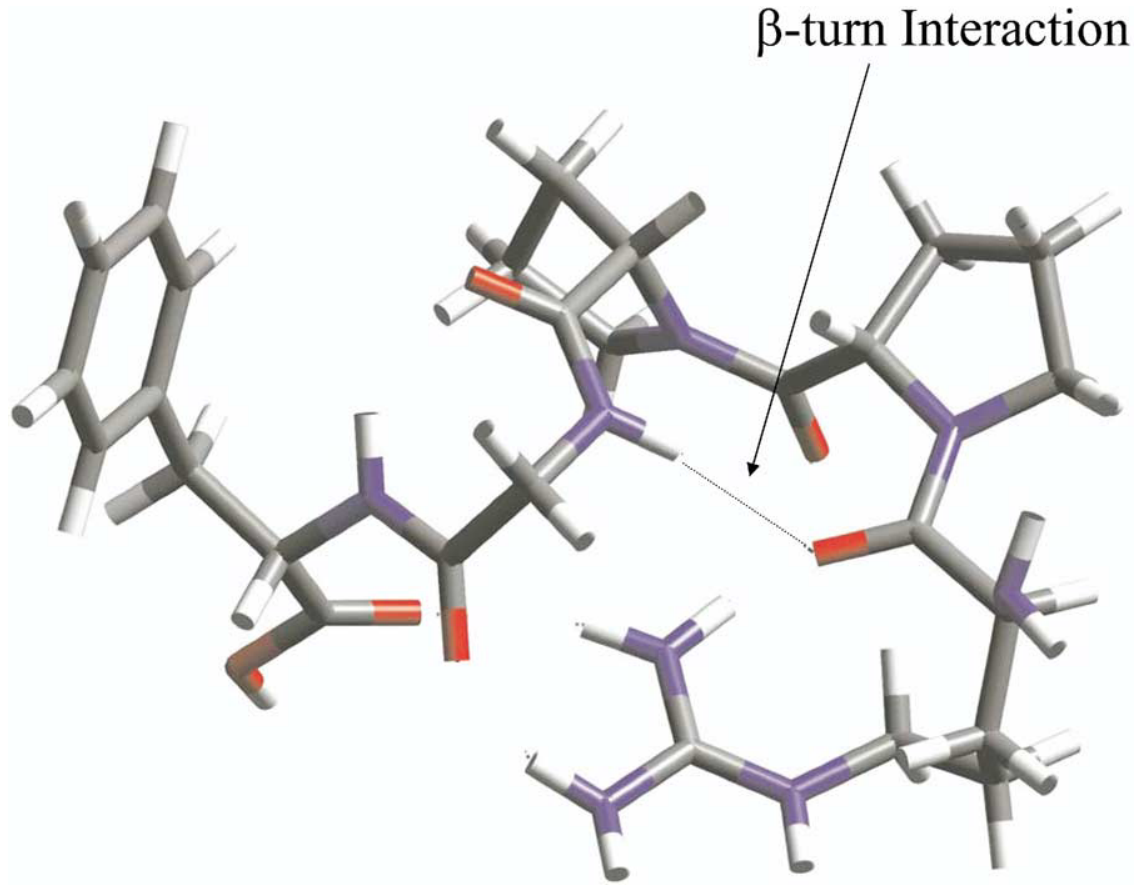

Figure 7. Proposed structure for $[\mathrm{M}+\mathrm{H}]^{+}$ions of BK1-5 ionized from organic solvent systems, having a collision-cross section of $163 \AA^{2}$.

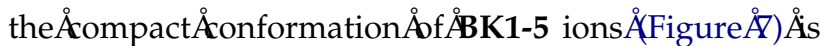
participating in hydrogen bonds with the C-terminus, which agrees with CD data for BK1-5 that suggest that the $\mathrm{N}$-terminal and $\mathrm{C}$-terminal portion of the molecule areÅn

In the proposed conformation $\left(\Omega_{\text {theo }}=168 \AA^{2}\right)$ for the second mobility peak observed in samples prepared usingÅaqueous Åsolvent $\AA ̊$ (Figure Å8), Åless Åhydrophilic groups interacting than was proposed for the compact conformation. More hydrophilic groups are exposed on the surface of the molecule, while the hydrophobic sites are turned to the inside of the molecule. Again, this assignment is consistent with NMR experiments on bradykinin that suggest that the major hydrogen bonding interactions present in aqueous solutions are those between Åhe hoptide Ånd Åhe Aolvent 28]. Addditionally, the charged guanidine group can interact with the phenylalanine side chain, and the carboxy terminus does not appear to interact strongly with the charge site. This assignment is consistent with NMR data of bradykinin in aqueous solutions that suggest that the arginine side chain is in the strongly asymmetric environment of

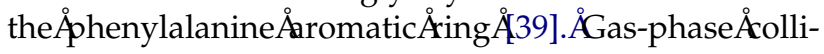
sion-induced decomposition (CID) data also suggest that $\arg ^{1} \mathrm{pro}^{2}$ interact with pro ${ }^{3} \mathrm{gly}^{4} \mathrm{phe}^{5}$ in such a way that it is believed that the interaction is occurring between $\arg ^{1}$ and phe $^{5}$ or pro $^{3}$ in both bradykinin and BK1-5 [10].

The pro $^{2}-$ pro $^{3}$ bond in this structure is in the trans configuration, and this agrees with results from solution-phase studies, which indicate that this is the pre-

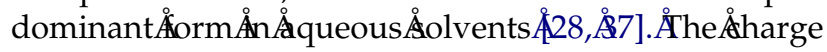

site in this conformation is in close proximity to the phenylalanine side chain as well as carbonyl oxygens, resulting in strong solvation of the basic site. We expect that such an interaction would reduce the rate and extent of $\mathrm{H} / \mathrm{D}$ exchange attributable to a reduction in the accessibility of the charge site to the in-coming neutral molecule. This proposed structure is in agreement with the gas-phase IM-MS and H/D exchange data presented in this paper as well as with extensive solution-phase CD and NMR data on bradykinin.

Very few interactions are occurring in the structure proposed for the low abundance extended conformation $\left(\Omega_{\text {theo }}=177 \AA^{2}\right)$ observed from aqueous solutions. The ̊structure $\AA$ shown $\AA$ in $\AA$ Figure $9 \AA$ Åcontains $\AA$ trans pro $^{2}$ - pro $^{3}$ bonding, in agreement with solution-phase

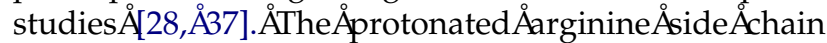
appears to be "solvated" by $\arg ^{1}$, pro $^{2}$, gly $^{4}$, and phe ${ }^{5}$, but the majority of the molecule does not participate in particular hydrogen bonding interactions. The lack of extensive intra-molecular interactions in this structure is not surprising considering that solution-phase $C D$ data also indicates no significant intra-molecular hydro-

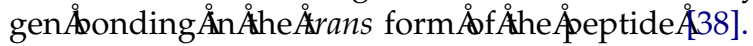

\section{Conclusions}

IM-MS data for BK 1-5 clearly shows that the number and cross-sections of conformers formed by MALD are highly dependent on the amount of methanol present in the solution from which the sample is deposited. IM-MS and gas-phase $\mathrm{H} / \mathrm{D}$ exchange data suggests that a more compact conformer is favored for mostly organic solu- 


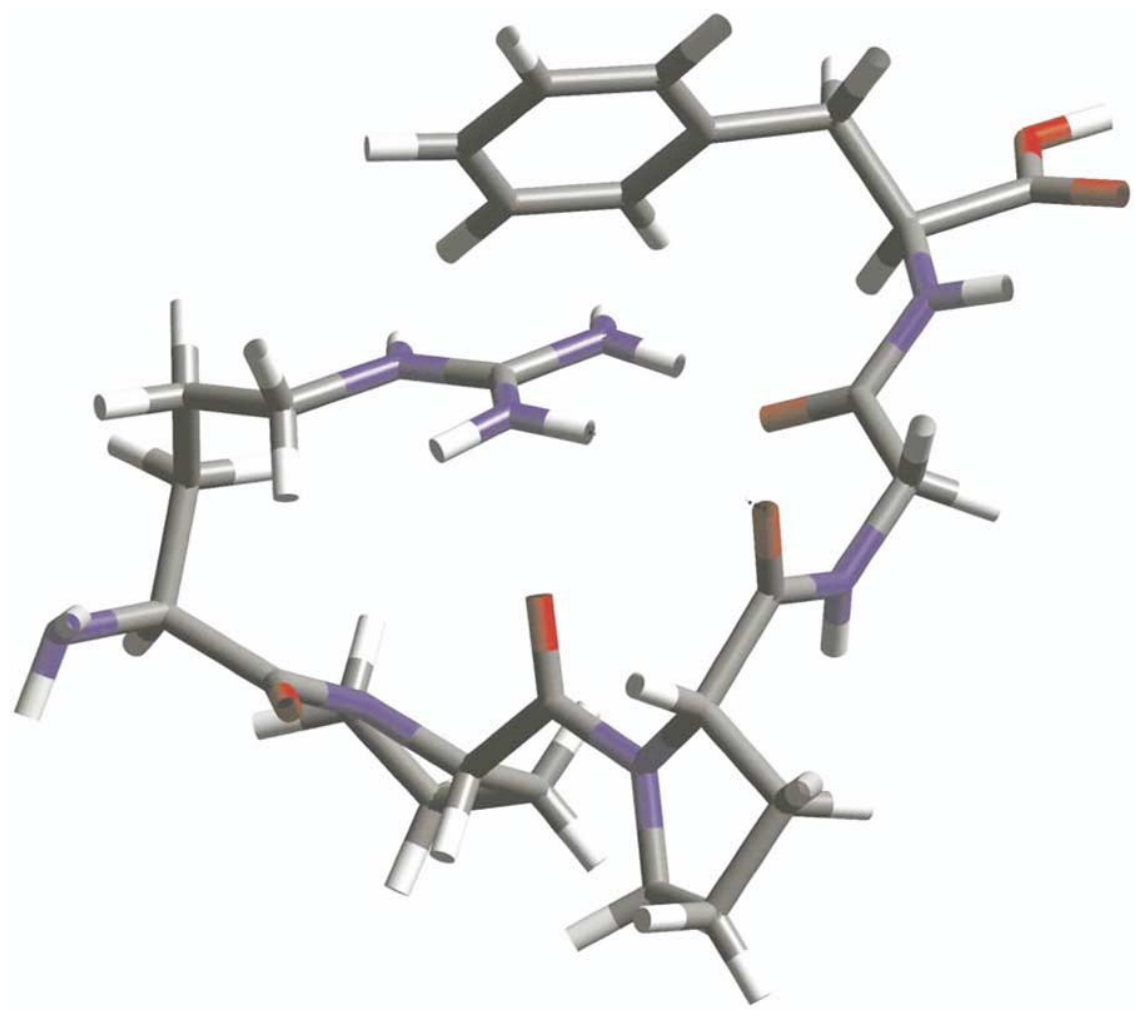

Figure 8. Proposed structure for $[\mathrm{M}+\mathrm{H}]^{+}$ions of BK1-5 ionized from aqueous solvent systems, having a collision-cross section of $168 \AA^{2}$.

tions, whereas at least one, and perhaps two, extended conformers are formed from aqueous solutions. Ion structures generated from MD based on IM collision cross-section measurements are quite similar to solution-phase structures derived from circular dichroism (CD) and NMR studies of bradykinin. Correlations between the solution-phase (CD and NMR) studies of bradykinin and its analogs, gas-phase IM-MS and H/D exchange data, and MD suggests that conformers of gas-phase BK1-5 $[\mathrm{M}+\mathrm{H}]^{+}$ions are dependent on the solvent composition and the MALDI sample. This conclusion is further supported by comparisons of IM-MS ATD of BK1-5 $[\mathrm{M}+\mathrm{H}]^{+}$ion obtained by conventional solid MALDI and MALDI from liquid matrices that have similar physical properties to the solvent used to prepare the solid matrix. Clearly, the combined results suggest that the solution-phase structure of BK1-5 is conserved during the MALDI process.

It is also interesting to note that our interpretation of the IM-MS and H/D exchange data is consistent with prior solution phase $\mathrm{H} / \mathrm{D}$ exchange experiments. In a previous study, we examined the solution-phase H/D exchange of bradykinin as a function of its solvent

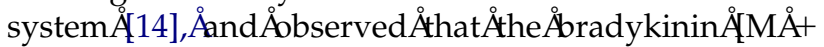
$\mathrm{H}]^{+}$ions formed by MALDI incorporate deuterium atoms as percentage of $\mathrm{D}_{2} \mathrm{O}$ added to the organic solvent increases. We suggested that bradykinin [M + $\mathrm{H}]^{+}$ions have a more compact conformation when formed from samples prepared using organic solvents

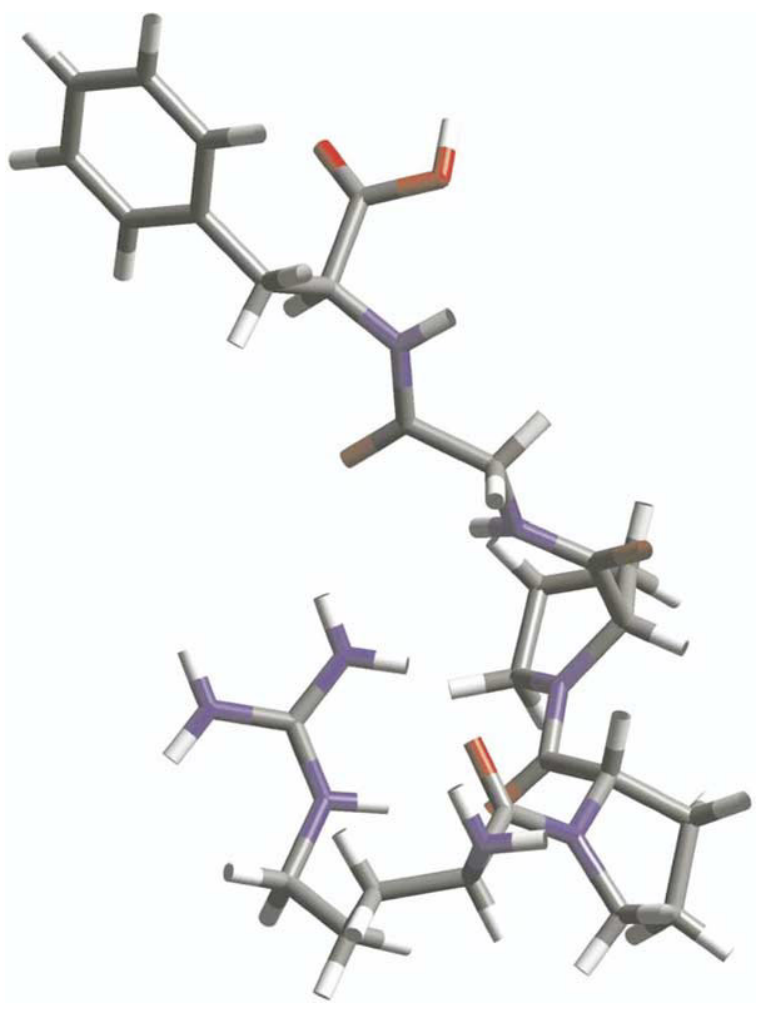

Figure 9. Proposed structure for $[\mathrm{M}+\mathrm{H}]^{+}$ions of BK1-5 ionized from aqueous solvent systems, having a collision-cross section of $177 \AA^{2}$. 
than from aqueous solvents, and because the compact peptide is less accessible to the deuterated solvent, which reduces the efficiency of H/D exchange. As water was added to the solution the conformation becomes more extended, which increases solvent accessibility and greater amounts of H/D exchange. The IM-MS and H/D exchange studies clearly illustrate the importance of intra-molecular interactions in stabilization of ion structure. In addition, comparisons of results from gas- and solution-phase experiments further illustrate the influence of solution-phase (MALDI sample preparation)

\section{Acknowledgments}

This research is supported by grants from the Robert A. Welch Foundation (A-1176) and the National Institutes of Health (1 R01 RR019587).

\section{References}

1. Ruotolo, B. T.; Verbeck, G. F.; Thomson, L. M.; Gillig, K. J.; Russell, D. H. Observation of Conserved Solution-Phase Secondary Structure in Gas-Phase Tryptic Peptides. J. Am. Chem. Soc. 2002, 124, 4214-4215.

2. Wang, J.; Cassady, C. J. Effects of Disulfide Linkages on Gas-Phase Reactions of Small Multiply Charged Peptide Ions. Int. J. Mass Spectrom. 1999, 182/183, 233-241.

3. Hudgins, Robert R.; Woenchkhaus, J.; Jarrold, M. F. High Resolution Ion Mobility Measurements for Gas Phase Proteins: Correlation Between Solution Phase and Gas Phase Conformations. Int. J. Mass Spectrom. Ion Processes 1997, 66, 497-507.

4. Counterman, A. E.; Clemmer, D. E. Cis-Trans Signatures of Proline-Containing Tryptic Peptides in the Gas Phase. Anal. Chem. 2002, 74, 1946-1951.

5. Wyttenbach, T.; von Helden, G.; Bowers, M. T. Gas-Phase Conformation of Biological Molecules: Bradykinin. J. Am. Chem. Soc. 1996, 118, 8355-8364.

6. Pace, C. N.; Treviño, S.; Prabhakaran, E.; Scholtz, J. M. Protein Structure, Stability, and Solubility in Water and Other Solvents. Phil. Trans. R. Soc. Lond. B 2004, 359, 1225-1235.

7. Price, W. D.; Jockusch, R. A.; Williams, E. R. Is Arginine a Zwitterion in the Gas Phase? J. Am. Chem. Soc. 1997, 119, 11988-11989.

8. Jockusch, R. A.; Lemoff, A. S.; Williams, E. R. Hydration of Valine-Cation Complexes in the Gas Phase: On the Number of Water Molecules Necessary to Form a Zwitterion. J. Phys. Chem. A 2001, 105, 10929-10942.

9. Gimon-Kinsel, M. E.; Barbacci, D. C.; Russell, D. H. Conformations of Protonated Gas-Phase Bradykinin Ions: Evidence for Intramolecular Hydrogen Bonding. J. Mass Spectrom. 1999, $34,124-136$.

10. Neuman, G. M.; Derrick, P. J. Intrinsic Chemistry of Peptide Ions: Field Desorption and Collision-Induced Decomposition of Bradykinin. Aus. J. Chem. 1984, 37, 2261-2277.

11. Freitas, M. A.; Marshall, A. G. Rate and Extend of Gas-Phase Hydrogen/Deuterium Exchange of Bradykinins: Evidence for Peptide Zwitterions in the Gas-Phase. Int. J. Mass Spectrom. 1999, 182/183, 222-231.

12. Schnier, P. D.; Price, W. D.; Jockusch, R. A.; Williams, E. R. Blackbody Infrared Radiative Dissociation of Bradykinin and Its Analogues: Energetics, Dynamics, and Evidence for SaltBridge Structures in the Gas Phase. J. Am. Chem. Soc. 1996, 118, 7178-7189.
13. Schaaf, T. G.; Stephenson, J. L.; McLuckey, S. A. The Reactivity of Gaseous Ions of Bradykinin and Its Analogues with Hydroand Deuteroiodic Acid. J. Am. Chem. Soc. 1999, 121, 8907-8919.

14. Figueroa, I. D.; Russell, D. H. Matrix-Assisted Laser Desorption Ionization Hydrogen/Deuterium Exchange Studies to Probe Peptide Conformational Changes. J. Am. Soc. Mass Spectrom. 1999, 10, 719-731.

15. Lee, S. C.; Russell, A. F.; Laidig, W. D. Three-Dimensional Structure of Bradykinin in SDS Micelles. Study Using Nuclear Magnetic Resonance, Distance Geometry, and Restrained Molecular Mechanics and Dynamics. Int. J. Pep. Protein Res. 1990, 35, 367-377.

16. Gillig, K. J.; Ruotolo, B.; Stone, E. G.; Russell, D. H.; Fuhrer, K.; Gonin, M.; Schultz, A. J. Coupling High-Pressure MALDI with Ion Mobility/Orthogonal Time-of-Flight Mass Spectrometry. Anal. Chem. 2000, 72, 3965-3971.

17. Stone, E. G. Development of a MALDI-Ion Mobility-SurfaceInduced Dissociation-Time-of-flight Mass Spectrometer for the Analysis of Peptides and Proteins. Ph.D. Thesis, Texas A and M University, College Station, 2003, pp 66-90.

18. Mason, E. A.; McDaniel, E. W. Transport Properties of Ions in Gases. John Wiley and Sons: New York, NY, 1988. pp 441-444.

19. Ruotolo, B. T.; Tate, C. C.; Russell, D. H. Ion Mobility-Mass Spectrometry Applied to Cyclic Peptide Analysis: Conformational Preferences of Gramicidin S and Linear Analogs in the Gas Phase. J. Am. Soc.Mass Spectrom. 2004, 15, 870-878.

20. Stone, E. G.; Gillig, K. J.; Ruotolo, B. T.; Russell, D. H. Optimization of a Matrix-Assisted Laser Desorption Ionization-Ion Mobility-Surface-Induced Dissociation-OrthogonalTime-of-Flight Mass Spectrometer: Simultaneous Acquisition of Multiple Correlated $\mathrm{MS}^{1}$ and $\mathrm{MS}^{2}$ Spectra. Int. J. Mass Spectrom. 2001, 212, 519-533.

21. Marini, J. T. Development and Implementation of a FT-ICR Mass Spectrometer for the Investigation of Ion Conformations of Peptide Sequence Isomers Containing Basic Amino Acid Residues by GasPhase Hydrogen/Deuterium Exchange. Ph.D. Thesis, Texas A and M University, College Station, 2003, pp 108-117.

22. Kirkpatrick, S.; Gelatt, C. D.; Vechhi, M. P. Optimization by Simulated Annealing. Science 1983, 220, 671-680.

23. Wilson, S. R.; Cui, W. Applications of Simulated Annealing to Peptides. Biopolymers 1990, 29, 225-235.

24. Mesleh, M. F.; Hunter, J. M.; Shvartsburg, A. A.; Schatz, G. C.; Jarrold, M. F. Structural Information from Ion Mobility Measurements: Effects of the Long Range Potential. J. Phys. Chem. 1996, 100, 16082-16086.

25. Shvartsburg, A. A.; Jarrold, M. F. An Exact Hard-Spheres Scattering Model for the Mobilities of Polyatomic Ions. Chem. Phys. Lett. 1996, 261, 86-91.

26. Williams, T. L.; Fenselau, C. p-Nitroaniline/Glycerol: A Binary Liquid Matrix for Matrix-Assisted Laser Desorption/ Ionization Analysis. Eur. Mass Spectrom. 1998, 4, 379-383.

27. Koster, C.; Castoro, J. A.; Wilkins, C. L. High-Resolution Matrix-Assisted Laser Desorption/Ionization of Biomolecules by Fourier Transform Mass Spectrometry. J. Am. Chem. Soc. 1992, 114, 7572-7574.

28. London, R. E.; Stewart, J. M.; Cann, J. R.; Matwiyoff, N. A. ${ }^{13} \mathrm{C}$ and ${ }^{1} \mathrm{H}$ Nuclear Magnetic Resonance Studies of Bradykinin and Selected Peptide Fragments. Biochemistry 1978, 17, 2270 2277.

29. Campbell, S.; Rodgers, M. T.; Marzluff, E. M.; Beauchamp, J. L. Structural and Energetic Constraints on Gas Phase Hydrogen/ Deuterium Exchange Reactions of Protonated Peptides with $\mathrm{D}_{2} \mathrm{O}, \mathrm{CD}_{3} \mathrm{OD}, \mathrm{CD}_{3} \mathrm{CO}_{2} \mathrm{D}$, and $\mathrm{ND}_{3}$. J. Am. Chem. Soc. 1994, 116, 9765-9766.

30. Reyzer, M. L.; Brodbelt, J. S. Gas-Phase H/D Exchange Reactions of Polyamine Complexes: $[\mathrm{M}+\mathrm{H}]^{+},[\mathrm{M}+$ alkali 
metal $]^{+}$, and $[\mathrm{M}+2 \mathrm{H}]^{2+}$. J. Am. Soc. Mass Spectrom. 2000, 11, 711-721.

31. Wyttenbach, T.; Bowers, M. T. Gas Phase Conformations of Biological Molecules: The Hydrogen Deuterium Exchange Mechanism. J. Am. Soc. Mass Spectrom. 1999, 10, 9-14.

32. Counterman, A. E.; Valentine, S. J.; Srebalus, C. A.; Henderson, S. C.; Hoaglund, C. S.; Clemmer, D. E. High-Order Structure and Dissociation of Gaseous Peptide Aggregates that are Hidden in Mass Spectra. J. Am. Soc. Mass Spectrom. 1998, 9, 743-759.

33. Cann, J. R.; Liu, X.; Stewart, J. M.; Gera, L.; Kotovych, G. A CD and an NMR Study of Multiple Bradykinin Conformations in Aqueous Trifluoroethanol Solutions. Biopolymers 1994, 34, 869878.

34. Cann, J. R.; Stewart, J. M.; Matsueda, G. R. A Circular Dichroism Study of the Secondary Structure of Bradykinin. Biochemistry 1973, 12, 3780-3788.
35. London, R. E.; Matwiyoff, N. A.; Stewart, J. M.; Cann, J. R. ${ }^{13} \mathrm{C}$ Nuclear Magnetic Resonance Study of the Cis-Trans Isomerism in X-Pro-Pro Tripeptides. Biochemistry 1978, 17, 2277-2283.

36. Lintner, K.; Fermandjian, S.; Regoli, D. Conformational Features of Bradykinin. Biochimie 1979, 61, 87-92.

37. Denys, L.; Bothner-By, A. A.; Fisher, G. H.; Ryan, J. W. Conformational Diversity of Bradykinin in Aqueous Solution. Biochemistry 1982, 21, 6531-6536.

38. Cann, J. R.; Stewart, J. M.; London, R. E.; Matwiyoff, N. On the Solution Conformation of Bradykinin and Certain Fragments. Biochemistry 1976, 15, 498-504.

39. Ruotolo, B. T., Russell, D. H. Gas-Phase Conformations of Proteolytically Derived Protein Fragments: Influence of Solvent on Peptide Conformation. J. Phys. Chem. B 2004, 108, 15321-15331. 